Portland State University

PDXScholar

$10-2016$

\title{
Förster Resonance Energy Transfer Between Molecules in the Vicinity of Graphene- Coated Nanoparticles
}

Tingting Bian

Beijing Union University

Railing Chang

National Taiwan Ocean University

Pui T. Leung

Portland State University, hopl@pdx.edu

Follow this and additional works at: https://pdxscholar.library.pdx.edu/phy_fac

Part of the Physics Commons

Let us know how access to this document benefits you.

\section{Citation Details}

Bian, Tingting; Chang, Railing; and Leung, Pui T., "Förster Resonance Energy Transfer Between Molecules in the Vicinity of Graphene- Coated Nanoparticles" (2016). Physics Faculty Publications and Presentations. 247.

https://pdxscholar.library.pdx.edu/phy_fac/247

This Post-Print is brought to you for free and open access. It has been accepted for inclusion in Physics Faculty Publications and Presentations by an authorized administrator of PDXScholar. Please contact us if we can make this document more accessible: pdxscholar@pdx.edu. 
Förster resonance energy transfer between molecules in the vicinity of graphenecoated nanoparticles

\author{
Tingting Bian ${ }^{1 *}$, Railing Chang ${ }^{2}$ and P. T. Leung ${ }^{3}$ \\ ${ }^{1}$ Institutue of Applied Science, Department of Foundational Science, \\ Beijing Union University, Chao Yang District, \\ Beijing, P. R. China, 100101 \\ ${ }^{2}$ Institute of Optoelectronic Sciences, National Taiwan Ocean University, Keelung, \\ Taiwan, R.O.C. \\ ${ }^{3}$ Department of Physics, Portland State University, P. O. Box 751, \\ Portland, OR 97207, U.S. A.
}

\begin{abstract}
The recent demonstration of the plasmonic enhanced Förster resonance energy transfer (FRET) between two molecules in the vicinity of planar graphene monolayers is further investigated using graphene-coated nanoparticles (GNP). Due to the flexibility of these nanostructures in terms of their geometric (size) and dielectric (e.g. core material) properties, greater tunability of the FRET enhancement can be achieved employing the localized surface plasmons. It is found that while the typical characteristic graphene plasmonic enhancements are manifested from using these GNP's, even higher enhancements can be possible via doping and manipulating the core materials. In addition, the broadband characteristics is further expanded by the closely-spaced multipolar plasmon resonances of the GNP's.
\end{abstract}

Key Words: graphene plasmonics; Förster resonance energy transfer (FRET)

* Corresponding author, email at: ting8386@126.com 


\section{Introduction}

As is well-known, it is of fundamental importance to understand how energy is transferred from an excited molecule (the donor, D) to a nearby molecule in its ground state (the acceptor, A) --- a process underlying many important photophysical and photochemical phenomena such as photosynthesis. Of the several possible

electromagnetic transfer mechanisms, the dominating one for distances $\left(R_{A D}\right)$ between $\mathrm{D}$ and $\mathrm{A}$ of a few $(\sim 2-6)$ nanometers is known as Förster resonance energy transfer (FRET) which depends mainly on the electrostatic dipole-dipole interaction between the two molecules. ${ }^{1}$ This FRET process is of particular relevance to many biological applications such as in the fluorescing probing of biomolecules. Nevertheless, despite its significance, FRET is a weak second-order process which falls off as $R_{A D}^{-6}$. This thus leads to serious limitation on its various applications such as in the range of fluorescence probing in biological systems. Hence, there has been great effort of research in the past several decades looking for different mechanisms leading to the enhancement of the FRET process. Among these, plasmonic enhancements employing the strong resonant fields from the collective oscillation of the free electrons at a metal surface have been explored as early as in the 1980's and have been found to be promising both theoretically and experimentally. ${ }^{2-9}$

Until recently, plasmonic enhanced FRET has been studied limiting to the interaction between $\mathrm{D}$ and $\mathrm{A}$ in the vicinity of metallic planar surfaces or particulate structures, most notably those of a noble metal. However, since most metals have their plasmonic resonances at visible or UV frequencies whereas application of FRET for biological systems often falls to the infrared (IR) regime, ${ }^{10}$ systems with lower plasmon 
frequencies are desired for the enhancement of FRET for these applications. The recent surge in the research of graphene plasmonics has thus filled this gap, not only because of its lower plasmonic frequency relative to that of metals, but also for its low loss and tunability via simple doping and gating processes. ${ }^{11}$

Such possibility of enhanced FRET using graphene plasmonics has been clearly demonstrated in some recent studies, ${ }^{12,13}$ in which the authors have performed theoretical investigations and concluded that FRET enhancement of $10^{4}$ to $10^{6}$ fold can be obtained in the IR with the D and A located in the vicinity of a planar graphene monolayer. Furthermore, this graphene-enhanced FRET is of a broadband nature which means the resonance condition between the frequency of the $\mathrm{D}$ and $\mathrm{A}$ and that of the plasmons can be much relaxed. Together with the easy tunable nature of the graphene plasmons, such enhanced FRET will have distinct and complementary role compared to that from employing metallic plasmonics. ${ }^{14}$

In the present work, we propose to investigate further the possibility of grapheneenhanced FRET using graphene-coated nanoparticles (GNP). Besides being in parallel with what was studied in the case of enhanced FRET by metallic plasmons --- where both extended and localized surface plasmons respectively from planar $^{4,6,8}$ and particulate $e^{2,3,5,7-9}$ structures have been utilized; nanoparticles also are much more versatile in their flexibility in being linked to the various systems to be probed. This is especially relevant in the case of in vivo probing of biological systems. In addition, great enhancement is expected with using GNP since it has been established that graphene of reduced dimensionality will have its plasmonic interaction strength increased. ${ }^{11}$ Hence the understanding of plasmonic enhancement for FRET from GNP will be significant in view 
of these potential applications. In the following, we will first briefly review and clarify a simple model for treating the plasmonics of GNP and then apply it to study the possible enhanced FRET from using these nanoparticles.

\section{Theoretical model}

In a recent work by Christensen et al, ${ }^{15}$ the authors have derived the optical response of a graphene-coated nanosphere by accounting for the graphene monolayer shell as one of negligible thickness and with its optical properties characterized by the following isotropic and local (i.e. only frequency dependent) surface conductivity function: ${ }^{15,16}$

$$
\sigma(\omega)=\sigma_{\text {intra }}(\omega)+\sigma_{\text {inter }}(\omega)
$$

with each of the intraband and interband terms expressed in the following forms:

$$
\begin{aligned}
& \sigma_{\text {int } r a}=\left(\frac{e^{2}}{\pi \hbar}\right)\left(\frac{2 i k_{B} T}{\hbar \tilde{\omega}}\right) \ln \left[2 \cosh \left(\frac{\varepsilon_{F}}{2 k_{B} T}\right)\right], \\
& \sigma_{\text {inter }}=\left(\frac{e^{2}}{\pi \hbar}\right)\left[\left(\frac{\pi}{4}\right) H\left(\frac{1}{2} \hbar \omega\right)+i \hbar \tilde{\omega} \int_{0}^{\infty} d \varepsilon \frac{H(\varepsilon)-H\left(\frac{1}{2} \hbar \omega\right)}{\hbar^{2} \tilde{\omega}^{2}-4 \varepsilon^{2}}\right],
\end{aligned}
$$

where $\tilde{\omega} \equiv \omega+i \gamma_{g}$ with $\gamma_{g}$ being the optical loss rate of graphene, $\left(e^{2} / \pi \hbar\right)$ the quantum conductance, $k_{B} T$ the thermal energy, $\varepsilon_{F}$ the Fermi energy, and $H(\varepsilon)=\sinh \left(\varepsilon / k_{B} T\right) /\left[\cosh \left(\varepsilon_{F} / k_{B} T\right)+\cosh \left(\varepsilon / k_{B} T\right)\right]$ is the population difference between energies $\mp \varepsilon$ (see Ref. 15). Note also that although the results in (2) were originally established for planar graphene structures, its applicability to spherical geometry can be justified as long as the curvature is small satisfying $k_{F} R \gg 1$ where $k_{F}$ is the Fermi vector and $R$ the radius of the sphere ${ }^{15}$. This latter condition is assured in most of our numerical studies below (e.g. for $\varepsilon_{F}=0.2 \mathrm{eV}$ and $R=20 \mathrm{~nm}, k_{F} R \sim 6$ ). 
Hence, within this model, the fully dynamic optical response of such a coated sphere will be similar to that given by the Lorenz-Mie theory, ${ }^{17}$ except that modifications are required due to the breakdown of the boundary condition from the continuity of the tangential magnetic field. This occurs simply because of the emergence of a surface current originated from the conductivity in (1) in the form $\vec{K}=\sigma \vec{E}$ in the presence of an external optical field. Thus the formal electrodynamics of optical scattering from this graphenecoated sphere is identical to that from a non-neutral sphere with a surface layer of free charge. In fact, the modified Lorenz-Mie theory derived in Ref. 15 can easily be recognized to be identical to that first formulated by Bohren and Hunt in the 1970's for a charged sphere. ${ }^{18}$ In the long-wavelength limit, the electrodynamic theory in Refs. 15 and 18 has been found to be accounted for by the multipole polarizability of the coated (or charged) sphere using an "effective dielectric function" of the following form [in Gaussian units (Ref. 19)]:

$$
\bar{\varepsilon}_{\ell} \equiv \varepsilon_{S}+i \frac{(\ell+1) \tau}{\rho},
$$

where $\ell$ is the multipole index, $\varepsilon_{S}$ is the dielectric function of the uncoated (or uncharged) sphere, $\rho=k R$ the size parameter for the sphere of radius $R$, and $\tau=(4 \pi / c) \sigma$ accounts for the graphene coating or the surface charge in the case of the non-neutral sphere. To our knowledge, the result in (3) was first obtained for charged sphere in our recent work ${ }^{19}$ and was re-derived in Ref. 15. Since in our following study of enhanced FRET from graphenecoated sphere, all the relevant length parameters are small compared to the source emission wavelength, we shall hence adopt the long-wavelength theory with the response from the coated sphere accounted for by the following multipole polarizability ${ }^{15}{ }^{19}$ : 


$$
\alpha_{\ell}=\frac{\ell\left(\bar{\varepsilon}_{\ell}-\varepsilon_{h}\right)}{\ell\left(\bar{\varepsilon}_{\ell}+\varepsilon_{h}\right)+\varepsilon_{h}} R^{2 \ell+1},
$$

where $\varepsilon_{h}$ is the dielectric function of the host for the sphere.

To study FRET between a donor (D) and an acceptor (A) in the vicinity of the graphene-coated sphere described above, we apply the conventional dipole-dipole interaction theory in the presence of the sphere (Fig. 1) and obtain the following electrostatic interaction energy responsible for the energy transfer from D to A in the form:

$$
U_{A}=-\vec{\mu}_{A} \cdot \vec{E}_{A}=U_{A D}+U_{A D \text {, ind }},
$$

where the acceptor dipole moment interacts with both the fields from the donor dipole $\vec{\mu}_{D}$, together with that produced by the induced multipole moments of the coated sphere due to the presence of the donor. ${ }^{2,3,13}$ Assuming the donor to be fixed at the north pole with its dipole moment on the $(r, \theta)$ plane, i.e. $\mu_{D \phi}=0$ (Fig. 1), the field on the acceptor at an arbitrary position $\left(r_{A}, \theta_{A}, \phi_{A}\right)$ can be obtained by solving the electrostatic boundary value problem, ${ }^{2,3,7,9}$ with the results for the various components given by the following expressions in terms of the Legendre and Associated Legendre functions:

$$
\begin{aligned}
& \text { For } r_{A}<r_{D}: \\
& E_{A r}=\sum_{\ell} \frac{1}{r_{D}^{\ell+2}}\left[\ell r_{A}^{\ell-1}+\ell(\ell+1) \frac{R^{2 \ell+1}}{r_{A}^{\ell+2}} \alpha_{\ell}\right]\left[(\ell+1) \mu_{D r} P_{\ell}\left(\cos \theta_{A}\right)+\mu_{D \theta} P_{\ell, 1}\left(\cos \theta_{A}\right) \cos \phi_{A}\right] \\
& E_{A \theta}=\sum_{\ell} \frac{1}{r_{D}^{\ell+2}}\left[r_{A}^{\ell-1}-\ell \frac{R^{2 \ell+1}}{r_{A}^{\ell+2}} \alpha_{\ell}\right]\left\{(\ell+1) \mu_{D r} P_{\ell, 1}\left(\cos \theta_{A}\right)+\frac{1}{2} \mu_{D \theta}\left[P_{\ell, 2}\left(\cos \theta_{A}\right)-\ell(\ell+1) P_{\ell}\left(\cos \theta_{A}\right) \cos \phi_{A}\right]\right\}
\end{aligned}
$$




$$
\begin{aligned}
& \text { For } r_{A}>r_{D}: \\
& \begin{aligned}
E_{A r}=\sum_{\ell} \frac{(\ell+1)}{r_{A}^{\ell+2}}\left\{\left[r_{D}^{\ell-1}+(\ell+1) \frac{R^{2 \ell+1}}{r_{D}^{\ell+2}} \alpha_{\ell}\right] \ell \mu_{D r} P_{\ell}\left(\cos \theta_{A}\right)+\left[r_{D}^{\ell-1}-\ell \frac{R^{2 \ell+1}}{r_{D}^{\ell+2}} \alpha_{\ell}\right] \mu_{D \theta} P_{\ell, 1}\left(\cos \theta_{A}\right) \cos \phi_{A}\right\} \\
E_{A \theta}=-\sum_{\ell}\left\{\left[r_{D}^{\ell-1}+(\ell+1) \frac{R^{2 \ell+1}}{r_{D}^{\ell+2}} \alpha_{\ell}\right] \frac{\ell \mu_{D r}}{r_{A}^{\ell+2}} P_{\ell, 1}\left(\cos \theta_{A}\right)\right. \\
\left.\quad-\frac{1}{2}\left[r_{D}^{\ell-1}-\ell \frac{R^{2 \ell+1}}{r_{D}^{\ell+2}} \alpha_{\ell}\right] \frac{\mu_{D \theta}}{r_{A}^{\ell+2}}\left[P_{\ell, 2}\left(\cos \theta_{A}\right)-\ell(\ell+1) P_{\ell}\left(\cos \theta_{A}\right)\right] \cos \phi_{A}\right\}
\end{aligned}
\end{aligned}
$$

Note that for the special case when the acceptor is placed on the spherical surface with $r_{A}=r_{D}$ (but not at the donor site), the source terms (i.e. those independent of $\alpha_{\ell}$ ) must be replaced by the one calculated directly from Coulomb's law with $\vec{\mu}_{D}$ as the source dipole. Eqs. (5)-(7) thus give a complete account of the interaction energy responsible for FRET between D and A in the presence of the graphene coated sphere provided we use Eqs. (1)-(4) for the multipolar polarizability $\alpha_{\ell}$ appeared in Eqs. (6) and (7). Since the FRET rate obtained from lowest order perturbation theory (Fermi's golden rule) is proportional to the square of the interaction energy ${ }^{20}$, in order to quantify the effect of the graphene-coated sphere, we consider the following enhancement ratio, ${ }^{2,3}$

$$
\mathcal{A}=\left|\frac{U}{U_{0}}\right|^{2}
$$

where $U_{0}$ is the well-known dipole-dipole interaction energy between $\mathrm{D}$ and $\mathrm{A}$ in free space given by the following expression:

$$
U_{0}=\frac{\vec{\mu}_{A} \cdot \vec{\mu}_{D}}{\left|\vec{r}_{A}-\vec{r}_{D}\right|^{3}}-\frac{3 \vec{\mu}_{A} \cdot\left(\vec{r}_{A}-\vec{r}_{D}\right) \vec{\mu}_{D} \cdot\left(\vec{r}_{A}-\vec{r}_{D}\right)}{\left|\vec{r}_{A}-\vec{r}_{D}\right|^{5}}
$$

In the following, we shall study numerically Eqs. (5) and (8) for various graphene-coated nanoparticles (both dielectric and semiconducting particles as studied in Ref. 15) as a 
function of the relative location and orientation between the donor and the acceptor, and compare the results with those for enhanced FRET from using other systems such as metallic nanoparticles ${ }^{2,3,7,9}$ and planar graphene monolayers. ${ }^{12,13}$

\section{Numerical results and Discussion}

For simplicity, we shall simulate the D-A interaction with the donor dipole always fixed to be along the $\mathrm{z}$ direction (i.e. $\mu_{D \theta}=0$ ) and located at the north pole at a distance of $1 \mathrm{~nm}$ from the particle surface. Unless otherwise specified, the GNP will have a size fixed at a radius of $R=20 \mathrm{~nm}$ so that quantum size effects can be ignored. ${ }^{21}$ All calculations were performed at room temperature $(T=300 \mathrm{~K})$. First we would like to study FRET from a graphene-coated polystyrene particle $(\varepsilon=2.4)^{15}$ as a function of the acceptor location. Figure 2(a) shows a 2D plot of the interaction energy $U_{A}$ in Eq. (5) as a function of the donor emission frequency and the angular position of the acceptor. Here the acceptor is fixed at a radial distance $2 \mathrm{~nm}$ from the particle surface with its dipole moment parallel to that of the donor. The result shows clearly the strong resonance enhancement at the graphene plasmon frequency in the IR regime at acceptor positions both near the north pole (where the donor is located) and also near the south pole of the sphere. The latter arises from the high symmetry in the multipolar induction fields at such an acceptor position and has been observed before in systems with spherical shells $\mathrm{s}^{22}$. This is shown more clearly in Fig. $2 \mathrm{~b}$ where we plot $U_{A}$ as a function of the acceptor position with the donor emitting at the dipole plasmon resonance frequency of the graphene-coated particle. We also remark that there is no analogue with the "propagation length" dependence observed in case with the FRET from planar graphene ${ }^{13}$ since only 
localized plasmons are excited with the GNP. Besides these "pole-location dependence", Fig. 2(a) also shows the typical broadband enhancement from graphene ${ }^{13}$, which is illustrated explicitly in Fig. 3 where the enhancement ratio in Eq. (8) is plotted as a function of donor emission frequency, with the acceptor in Fig. 2 located at the south pole. We remark that these broadband and IR enhancements are characteristics of graphene plasmonics in contrast to that using metals, as noted previously in the work of Biehs and Agarwal. ${ }^{13}$ While this broadband enhancement is mainly originated from the linear dispersion of the Dirac electrons in graphene ${ }^{11}$, the graphene-coated particle contributes to this also from its large number of closely-spaced multipolar plasmon resonances. This feature is absent in the case of enhanced FRET from planar graphene stucture $^{12,13}$. In addition, we also compare the FRET spectrum from two GNP's of different sizes in Fig. 3, and it is clear that this extra tunability with greater enhancement via manipulation of the multipolar resonances is attainable simply by varying the dimension of the GNP's. Note that even for $R=10 \mathrm{~nm}$, quantum size effects are only marginally noticeable. ${ }^{21}$

Next we study the distance dependence of the GNP induced FRET, where we set the $\mathrm{D}$ and $\mathrm{A}$ dipoles to be parallel along $\mathrm{z}$ and located at the poles. Figure 4(a) shows the enhancement ratio as a function of the radial distance of the acceptor at the dipole plasmon frequency for different core materials as used in Ref. 15. Here the distance $r_{A}$ is measured from the GNP surface. It is clear that while the FRET enhancement decreases as the acceptor starts to move away from the GNP, the degree of enhancement depends crucially on the core material, with the "hollow graphene shell" yielding the highest possible enhancement. This can be understood since enhancement is doom to decrease 
when the core material is either dissipative (e.g. doped semiconductor) or polarizable (e.g. polystyrene), or both. Similar behavior in optical extinction from these GNP's had also been observed previously ${ }^{15}$. Hence the "unrealistic" case of a hollow graphene shell will in principle yield the highest FRET enhancement with thousand times possible even for the acceptor at a distance several times that of the particle radius from the GNP surface. Thus in real applications, core transparent materials of a low dielectric constant will be preferred for the fabrication of the GNP for FRET enhancement.

Moreover, for near field interaction as in the FRET case, it is well known that higher order plasmon resonances will yield even higher enhanced fields. Hence in Fig. 4(b) we compare results with the donor frequency fixed at several higher multipole resonances of the GNP for the hollow case and for the polystyrene core case, respectively. The results demonstrate clearly that at close distances the higher multipole resonance will lead to even higher FRET enhancement as anticipated.

One interesting issue was raised in the study with planar graphene ${ }^{13}$ when two mutually perpendicular dipoles with their relative position vector perpendicular to one of the dipoles can still undergo FRET in the presence of the graphene layer. To study this phenomenon in our GNP-induced FRET, we fix the acceptor at three different locations and calculate the interaction energy as a function of the acceptor orientation angle $\theta_{\mu A}$ with the results shown in Fig. 5. It is seen both at the south pole and at the equator, the D-A interaction is rather symmetric about $\theta_{\mu A}=90^{\circ}$, the position with A at $45^{\circ}$ oscillates with the values of $\theta_{\mu A}$ in a way "out of phase" relative to the two more symmetric positions. Furthermore, it is interesting to see that the "zero interaction" possibility reappears again even in the presence of the GNP at $\theta_{\mu \mathrm{A}} \sim 90^{\circ}$ when each of the D and A 
is located at the two separate poles of the sphere, just like the well-known situation with two dipoles interacting in vacuum. Note that in this case, a "cusp" at the zero interaction point arises because we are plotting the absolute values of the interaction energy which change sign at this point.

Following this we would like to demonstrate the tunability of the GNP enhanced FRET. It is well-established that the Fermi level $\varepsilon_{F}$ of graphene is tunable via doping and gating over a range of values up to $\sim 1 \mathrm{eV}$ in magnitude ${ }^{11}$, with each of the intraband and interband transitions responding in its own characteristic way. While the graphene plasmon frequency will go up $\sim \sqrt{\varepsilon_{F}}$, the interband transition will be suppressed via the Pauli blocking effect with the increase in the Fermi energy ${ }^{23,24}$. These effects are indeed manifested in our study on the GNP-induced FRET as revealed from Fig. 6, in which we have plotted the enhanced FRET spectrum for a graphene-coated polystyrene particle for different values of $\varepsilon_{F}$. It is clear that as $\varepsilon_{F}$ increases, more higher order plasmonic peaks appear with each blue-shifted yielding greater enhancements at the same time. This is a result from both the increase in the plasmon frequency and the decrease in overall damping due to the suppression of interband transitions. In fact, it is exciting to see that much higher FRET enhancements can be achieved via heavier doping and employing the higher multipole resonances of the GNP. In addition, Fig. 6(b) shows clearly the linear relation between the plasmonic resonance frequency (for each of the multipole resonance) and $\sqrt{\varepsilon_{F}}$ as expected ${ }^{24}$.

Finally, being inspired by the results from Fig. 4, we venture to quest for the possibility of even higher FRET enhancement to be achieved by exploiting gain medium as the core material for the GNP. Using the following dielectric function for a 
hypothetical gain medium ${ }^{25}: \varepsilon_{g}=\varepsilon_{B}+g \Gamma /\left(\omega-\omega_{r}+i \Gamma\right)$ which depends on the background dielectric constant, the gain level, the gain spectral width and the gain center frequency, respectively; we have shown in Fig. 7 it is possible to attain much higher FRET enhancement using graphene-coated particle with a gain medium core by choosing appropriately the relevant parameters in the gain dielectric function. Although such gaininduced enhancement (over the hollow graphene shell) is significant only by setting the gain frequency at the low IR range, the effect can still survive for a higher value with the gain frequency up to $1.0 \mathrm{eV}$. Though such low frequency gain material may not be too realistic at the present time, it remains a possibility with future technology for achieving extremely large enhanced IR FRET using GNP's with a gain medium core, and may become even more realistic if the graphene plasmon frequency is further increased via doping or gating.

\section{Conclusion}

Within a simple model, we have demonstrated in this work enhanced FRET at the IR regime is possible using graphene-coated particles, in complement to similar applications of metallic nanoparticles in the visible domain. As noted above, this GNP approach can provide greater flexibility than the planar graphene platform ${ }^{12,13}$ in real applications to probe biological systems in vivo, and can lead to highly-enhanced broadband FRET via doping and gating. In addition, such systems employing localized plasmons is not as sensitive as the extended ones (on planar systems) to any characteristic length parameter (e.g. the propagation length) of the system and hence less restricted in applications via implementation of these particles into the (biological) sample to be probed. Furthermore, the broadband characteristics from the Dirac charge carriers is 
further expanded due to the closely-spaced multipolar resonances in these GNP's. With the recent demonstration of the feasibility for the fabrication of such GNP's ${ }^{26}$, it is possible to achieve even higher enhancement of wider bandwidth via doping and employing certain gain medium which can possibly be realized with the advance in technology. One final remark on the limitation of our model is the negligence of the nonlocal response from the graphene coating ${ }^{15,27}$. While these effects are likely not very significant for the relatively large GNP size $(R=20 \mathrm{~nm})$ we have considered, they will possibly lead to relatively lower enhancements for cases with the molecules being very close to the GNP, but with most of the qualitative features reported above to prevail. We thus conclude that the GNP enhanced FRET as demonstrated theoretically in our work here is indeed feasible, and can lead to significant applications in the probing of biological systems with fluorophores at IR frequencies.

\section{Acknowledgments}

This work was supported by Beijing Talent Fund (Grant No. 2014000020124G061), as well as by the National Science Council of Taiwan through grant MOST 103-2112-M019 -003 -MY3. PTL thanks Prof. Hai-Pang Chiang for his hosting during his visit to NTOU. 


\section{References}

${ }^{1}$ T. Förster, Ann. Phys. 437, 55-75 (1948); Discuss. Faraday Soc. 27, 7-17 (1959).

${ }^{2}$ J. I. Gersten and A. Nitzan, Chem. Phys. Lett. 104, 31-37 (1984).

${ }^{3}$ X. M. Hua, J. I. Gersten, and A. Nitzan, J. Chem. Phys. 83, 3650-3659 (1985).

${ }^{4}$ P. Andrew and W. L. Barnes, Science 290, 785 (2000); ibid 306, 1002-1005 (2004).

${ }^{5}$ See, e.g., the following works by Lakowicz and coworkers: Annal. Biochem. 315, 160169 (2003); J. Fluoresc. 13, 69-77 (2003); J. Phys. Chem. C 111, 50-56 (2007); 11784$11792(2007)$.

${ }^{6}$ J. I. Gersten, Plasmonics 2, 65-77 (2007).

${ }^{7}$ H. Y. Xie, H. Y. Chung, P. T. Leung, and D. P. Tsai, Phys. Rev. B 80, 155448 (2009); H. Y. Chung, P. T. Leung, and D. P. Tsai, Plasmonics 5, 363-368 (2010); R. Chang, P. T. Leung, and D. P. Tsai, Opt. Express 22, 27451-27461 (2014).

${ }^{8}$ C. A. Marocico and J. Knoester, Phys. Rev. A 84, 053824 (2011).

${ }^{9}$ J. A. Gonzaga-Galeana and J. R. Zurita-Sanchez, J. Chem. Phys. 139, 244302 (2013).

${ }^{10}$ See, e.g., L. Xiong, A. J. Shuhendler, and J. Rao, Nat. Commun. 3, 1193 (2012).

${ }^{11}$ F. H. L. Koppens, D. E. Chang, and F. J. Garcia de Abajo, Nano Lett. 11, 3370-3377 (2011); F. J. Garcia de Abajo, ACS Photonics, 1, 135-152 (2014).

${ }^{12}$ K. A. Velizhanin and T. V. Shahbazyan, Phys. Rev. B 86, 245432 (2012).

${ }^{13}$ S. A. Biehs and G. S. Agarwal, Appl. Phys. Lett. 103, 243112 (2013).

${ }^{14}$ For an interesting comment on graphene versus metal plasmonics, see F. Xia, Nat. Photonics 7, 420 (2013).

${ }^{15}$ T. Christensen, A.-P Jauho, M. Wubs, and N. A. Mortensen, Phys. Rev. B 91, 125414 (2015). 
${ }^{16}$ L. A. Falkovsky and A. A. Varlamov, Eur. Phys. J. B 56, 281-284 (2007).

${ }^{17}$ L. Lorenz, Videnskab. Selskab. Skrifter 6, 1-62 (1892); G. Mie, Ann. Phys. 25, 377445 (1908).

${ }^{18}$ C. F. Bohren and A. J. Hunt, Can. J. Phys. 55, 1930-1935 (1977).

${ }^{19}$ H. Y. Chung, P. T. Leung, D. P. Tsai, J. Chem. Phys. 138, 224101 (2013). Note that Gaussian units have been used in this Reference and we have adopted the same unit system in the present work. The corresponding result for Eq. (3) in SI units can be found from Ref. [15]. Note also that all the numerical results obtained in this paper are independent of the unit system used in the theory.

${ }^{20}$ For a comment on the possible dependence on other factors such as the apparent quantum yield of the donor, see the paper by R. Chang et al cited in Ref. 7 .

${ }^{21}$ S. Thongrattanasiri, A. Manjavacas, and F. J. Garcia de Abajo, ACS Nano 6, 17661775 (2012).

${ }^{22}$ M. Durach, A. Rusina, V. I. Klimov, and M. I. Stockman, New J. Phys. 10, 105011 (2008); see also the paper by H. Y. Xie et al in Ref. 7.

${ }^{23}$ E.H. Hwang, S. Das Sarma, Phys. Rev. B 75, 205418 (2007).

${ }^{24}$ K. F. Mak, L. Ju, F. Wang, and T. F. Heinz, Solid State Commun. 152, 1341 (2012).

${ }^{25}$ A. Veltri and A. Aradian, Phys. Rev. B 85, 115429 (2012).

${ }^{26}$ H.Yang, Z. Hou, N. Zhou, B. He, J. Cao, andY.Kuang, Ceramics International 40, 13903 (2014).

${ }^{27}$ W. Wang and J. M. Klinaret, Phys. Rev. B 87, 195424 (2013). 


\section{Figure Captions (All figures being color online)}

1. Configuration of the problem.

2. Plot of the variation of interaction energy (absolute value) between the donor and the acceptor as a function of the angular position of the acceptor, with both dipole moment along the $\mathrm{z}$ direction and the donor fixed at the north pole at $1 \mathrm{~nm}$ from the GNP surface, while the acceptor moves on a spherical surface 2 nm away from the GNP surface: (a) a 2D plot with both the donor frequency and acceptor position varying and (b) with the donor frequency fixed close to resonance at $0.221 \mathrm{eV}$. The GNP radius is fixed at $20 \mathrm{~nm}$.

3. FRET enhancement spectrum with the radial distances as in Fig. 2 for two different sizes of the GNP. Both dipoles are along the $\mathrm{z}$ direction and fixed at the two poles.

4. FRET enhancement as a function of the radial distance of the acceptor with the dipoles as in Fig. 3. Different core materials for the GNP are considered and in each case the donor frequency is chosen to be the resonance for that particular structure. Fig. 4(a) is at the dipole resonance and 4(b) compares the results from several lowest multipole resonances. Note that $r_{A}$ is measured from the GNP surface in this figure.

5. Interaction energy as a function of the relative orientations between the two dipoles with the radial distances as in Fig. 2 and the acceptor at three different angular positions: $\pi / 4, \pi / 2$, and $\pi$. The donor is the same as in Fig. 2 but the GNP has a vacuum core. Note that the cusp arises due to that the absolute 
value of $U$ is plotted and $U$ changes sign for the case when both dipoles are at the poles.

6. Tunability of enhanced FRET with the variation of the Fermi energy of the GNP for the dipoles as in Fig. 3. Fig. 6(a) shows the FRET spectrum and Fig. 6(b) shows the linear relation between the various multipolar resonance frequency as a function of the square root of the Fermi energy.

7. Possibility for further FRET enhancement over the hollow GNP case with gain medium used as the core material. The values of the gain level is taken as $g=0.3$ and the gain spectral width is taken as 20 times of that of the optical loss rate of graphene (Ref. 15), while the gain frequency is chosen to be 0.4 and $1.0 \mathrm{eV}$, respectively. 


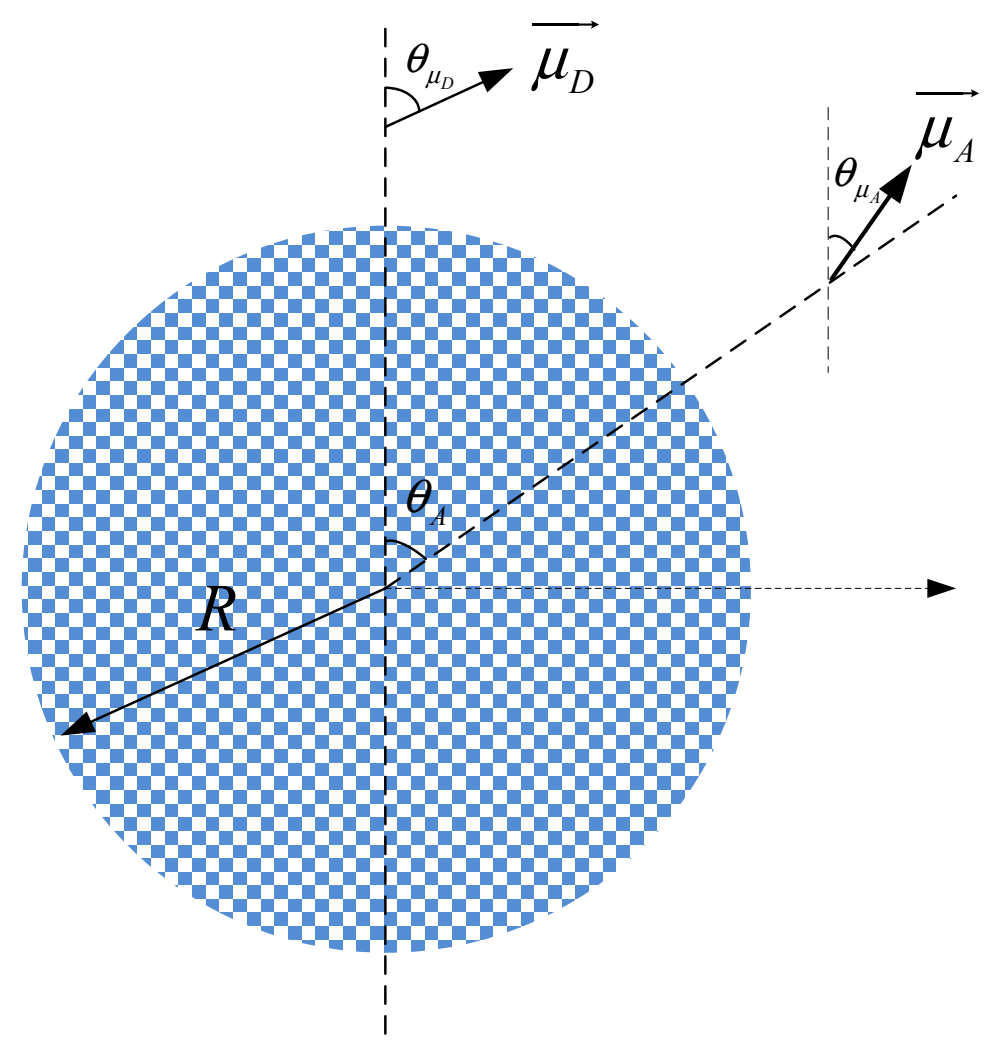

Fig. 1 

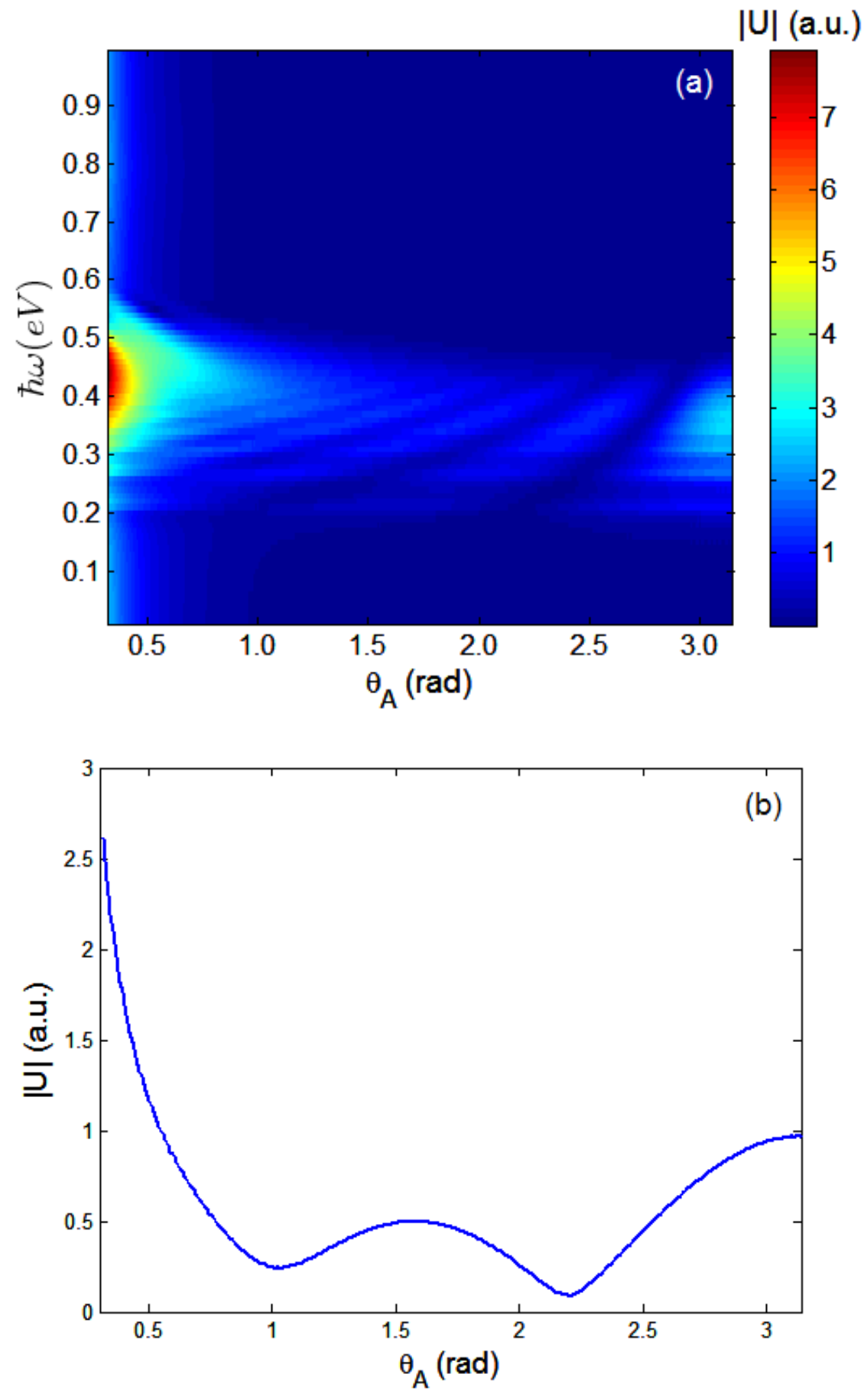

Fig. 2 


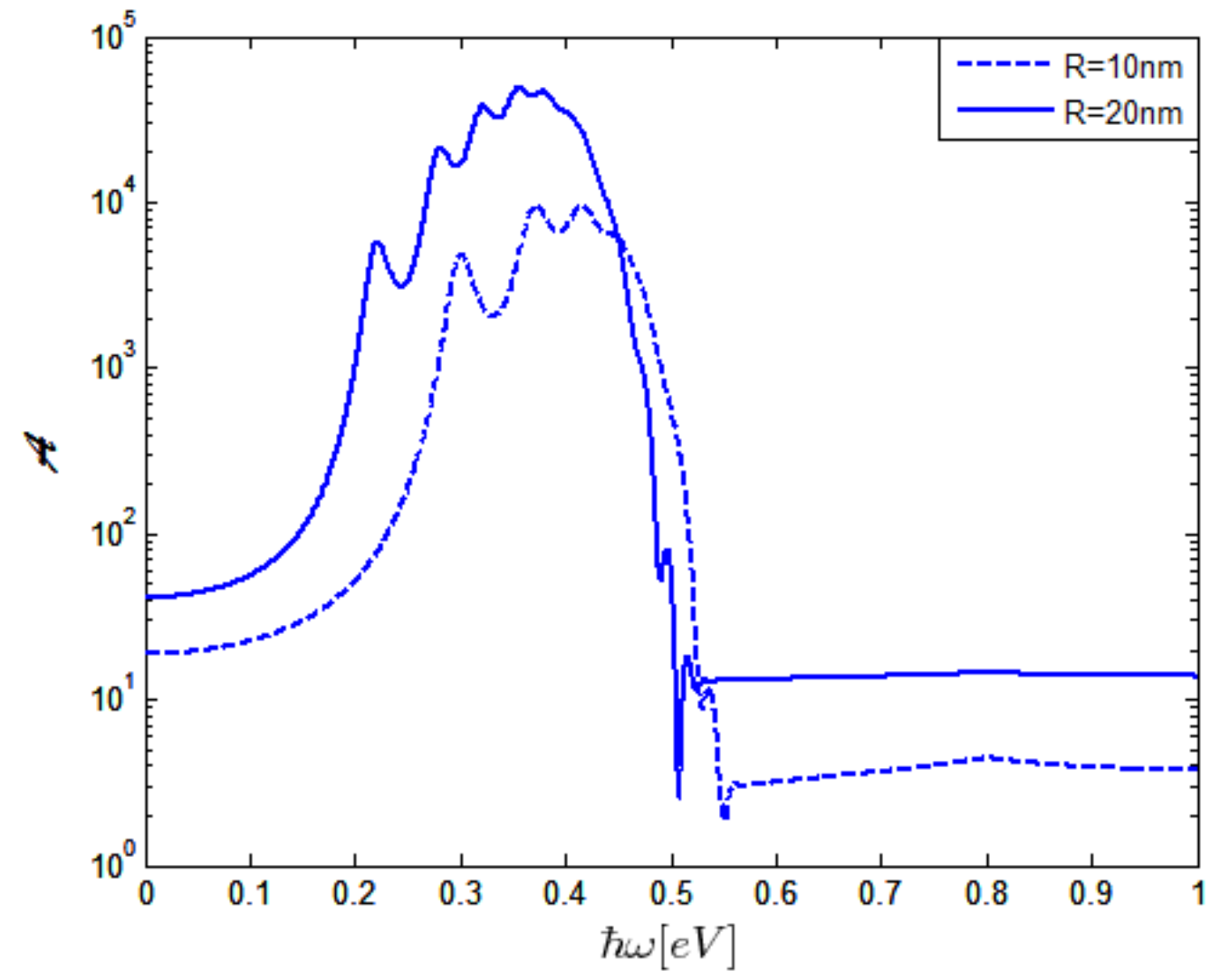

Fig. 3 

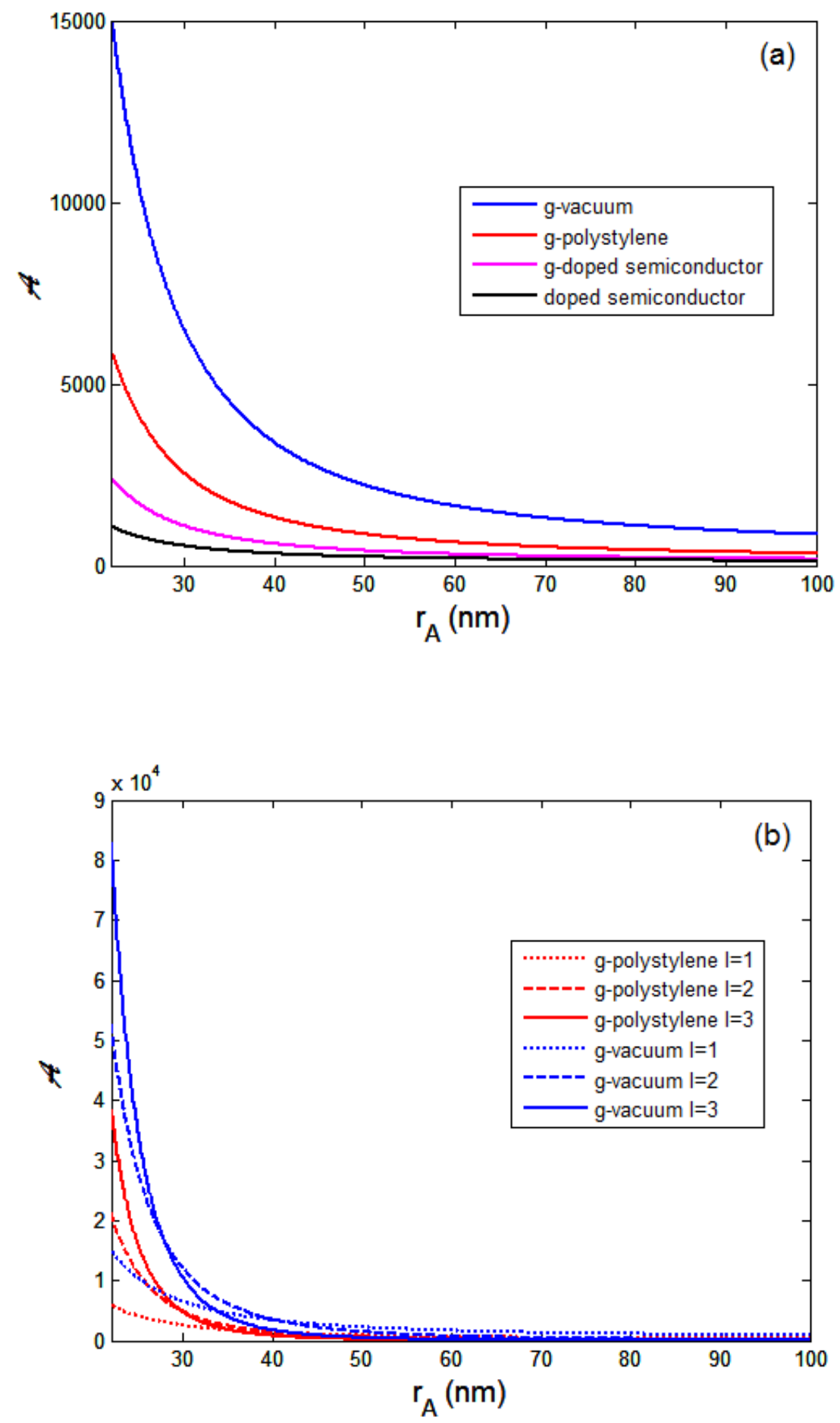

Fig. 4 


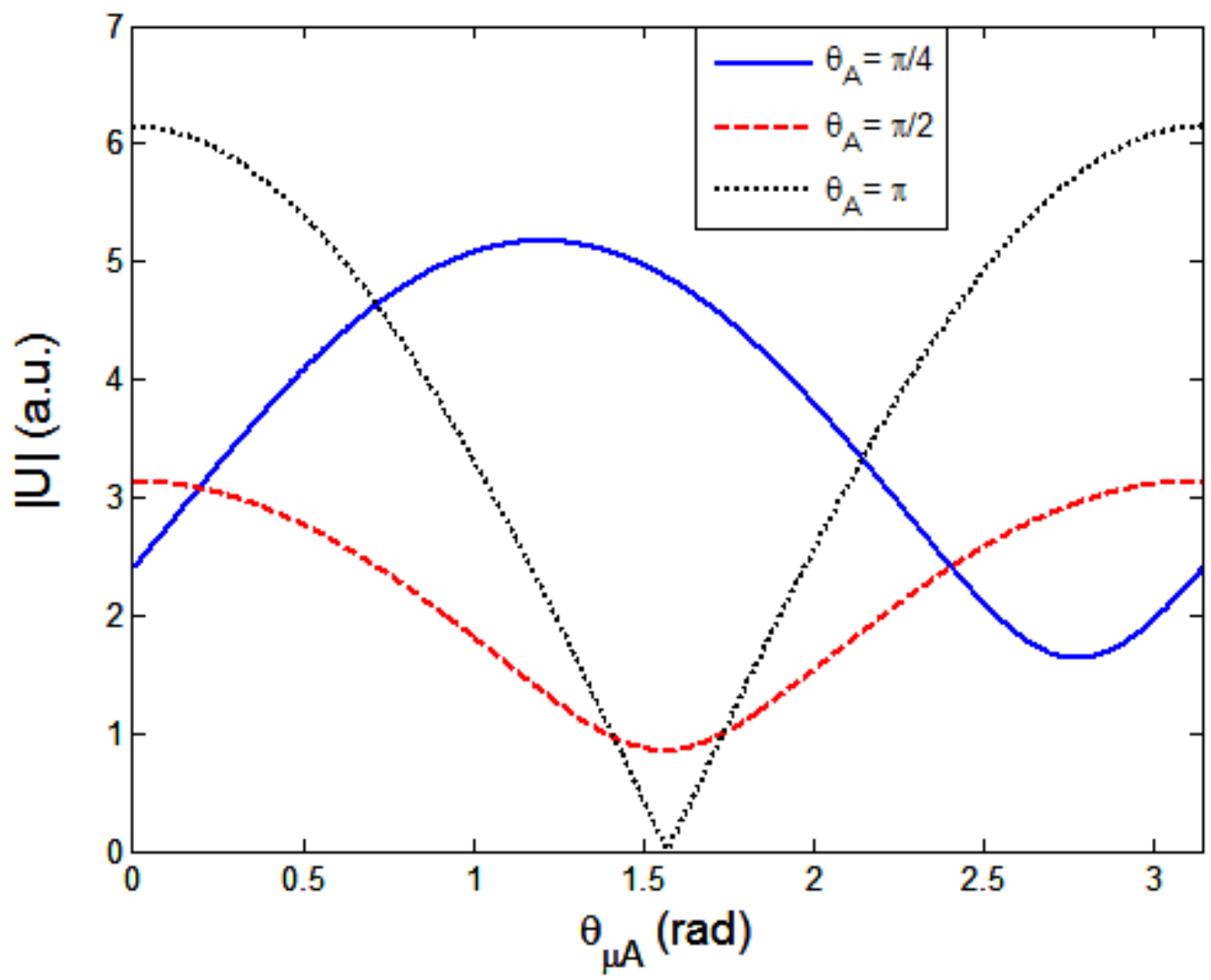

Fig. 5 

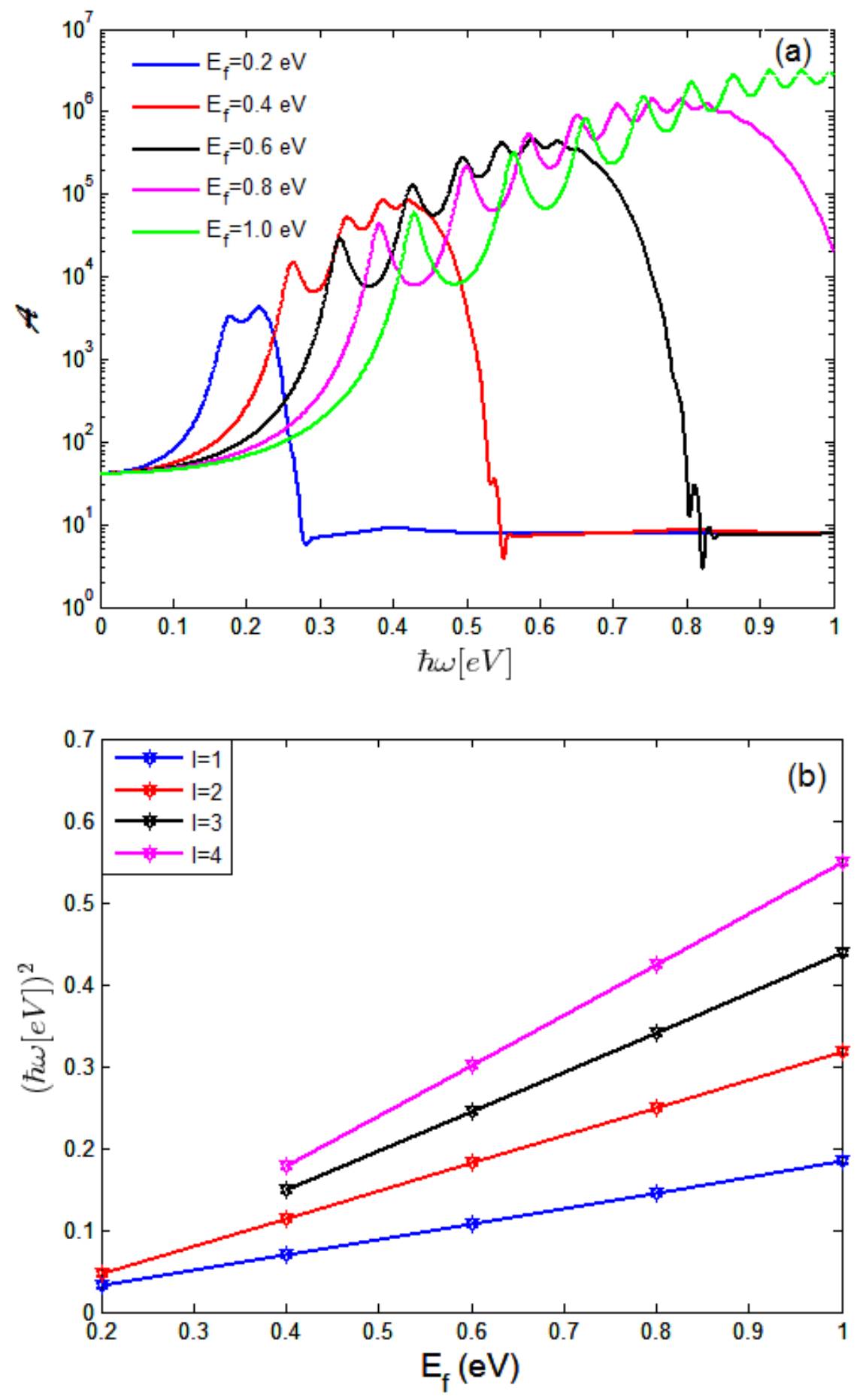

Fig. 6 


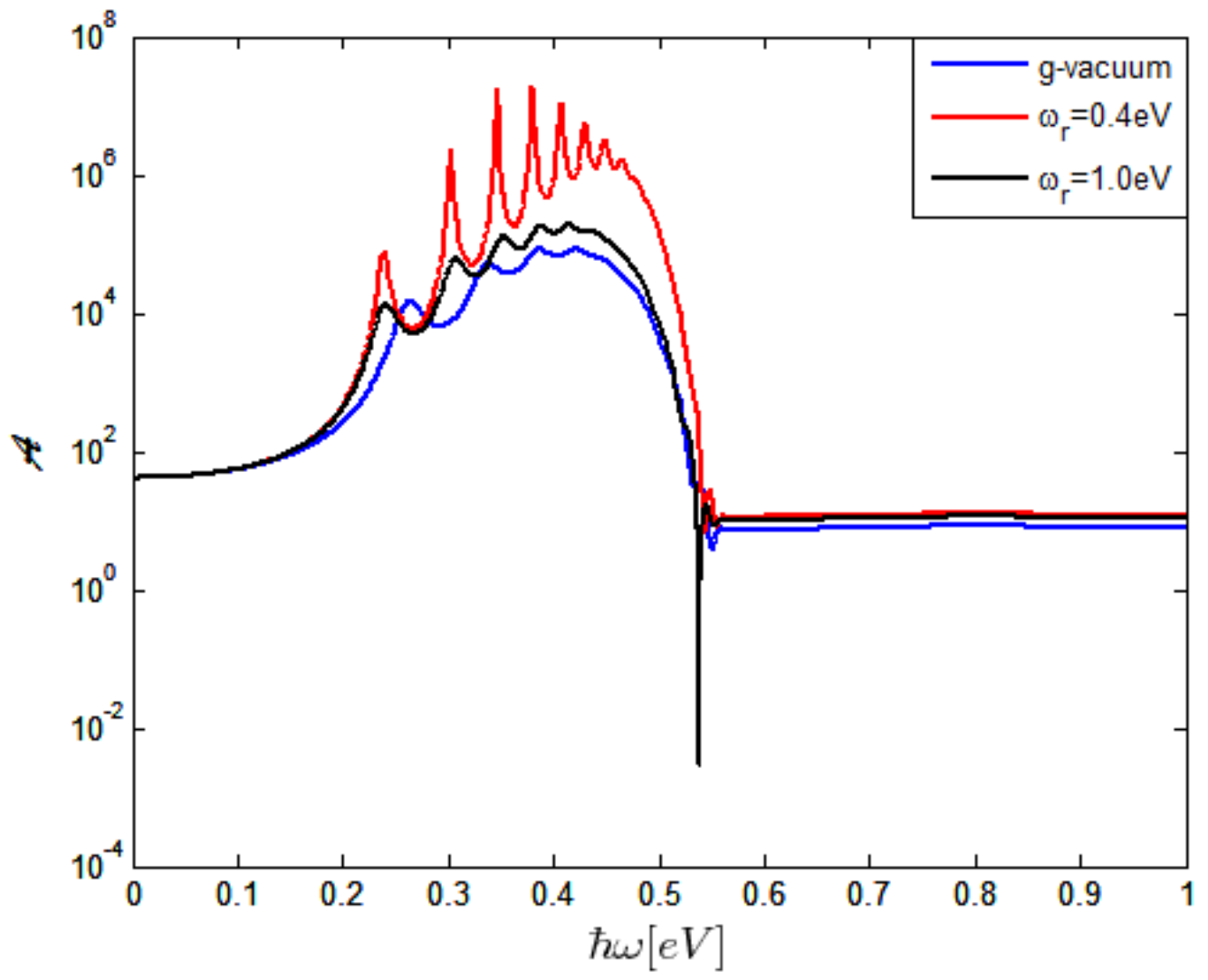

Fig. 7 